\title{
Levistilide A Ameliorates NLRP3 Expression Involving the Syk-p38/JNK Pathway and Peripheral Obliterans in Rats
}

\author{
Huining Guo, Li Sun, Shuang Ling $\mathbb{D}$, and Jin-Wen Xu $\mathbb{C}$ \\ Institute of Interdisciplinary Medical Science, Shanghai University of Traditional Chinese Medicine, Shanghai 201203, China \\ Correspondence should be addressed to Jin-Wen Xu; jinwen.xu88@gmail.com
}

Received 5 April 2018; Revised 23 June 2018; Accepted 19 July 2018; Published 12 August 2018

Academic Editor: Stefan C. Vesa

Copyright (C) 2018 Huining Guo et al. This is an open access article distributed under the Creative Commons Attribution License, which permits unrestricted use, distribution, and reproduction in any medium, provided the original work is properly cited.

\begin{abstract}
Background. Inflammation is one of the most important pathogeneses of thromboangiitis obliterans (TAO). The NLRP3 inflammasome plays a vital role in the body's immune response and disease development. It can be activated by numerous types of pathogens or danger signals. As the core of the inflammatory response, the NLRP3 inflammasome may provide a new target for the treatment of various inflammatory diseases. Levistilide A (LA) is a phthalide dimer isolated from umbelliferous plants. Its pharmacological effect is largely unknown. This study revealed the effects of LA on endothelial cell activation, NLRP3, IL-1 $\beta$, TNF- $\alpha$, IL-32, and CCL-2, VCAM-1, MCP-1, and the spleen tyrosine kinase (Syk)-p38/JNK signaling axis and its effect on vasculitis in rats. Results. LA inhibited endothelial activation and the expression of IL- $1 \beta$, TNF- $\alpha$, IL-32, CCL-2, VCAM-1, and MCP-1. LA directly obstructed Syk phosphorylation and activity in a dose-dependent manner, inhibited the activity of p38 and JNK, and reduced the expression of NLRP3 in human umbilical vein endothelial cells and vascular tissue of rats with vasculitis. Conclusion. LA suppressed NLRP3 gene expression by blocking the Syk-p38/JNK pathway and reduced damage to the rats' limbs in the thromboangiitis obliterans model.
\end{abstract}

\section{Introduction}

Vasculitis is an inflammatory cell infiltration around blood vessel walls and blood vessels, accompanied by vascular damage, cellulose deposition, fibrosis, and endothelial cell and myocyte necrosis. Vasculitis is characterized by systemic inflammation and endothelial injury [1]. Endothelial activation and injury are fundamental to the pathogenesis. Endothelial activation and injury increase expression of endothelial cell adhesion and cause endothelial switching to the prothrombotic phenotype. Thromboangiitis obliterans (TAO) or Buerger's disease (BD) is a thrombotic occlusive, nonatherosclerotic segmental inflammatory disease that causes ischemia in small and medium vessels in the extremities of the limbs. TAO is characterized by segmental thrombotic occlusions by highly mononuclear cellular thrombi. Endothelial dysfunction may play a crucial role in BD [2]. Patients with BD have a diminished capability for endothelium-dependent vasodilation and higher levels of some circulating markers of inflammation, such as leukocytes, C-reactive protein, and intercellular adhesion molecule-1 [3].

Levistilide A (LA) (Figure 1(a)) is a phthalide dimer isolated from Ligusticum chuanxiong and Angelica sinensis, both of them belonging to the Umbelliferae herb family [4-7]. These herbs are often used in oriental traditional medicinal formulas, such as Danggui-Shaoyao-San (DSS). At present, there are few reports on LA research. LA inhibits PDGF-BB-activated hepatic stellate cell proliferation; the mechanism may involve cell cycle inhibition and apoptosis mechanisms. LA might be a potential antifibrotic drug for the treatment and prevention of hepatic fibrosis [8]. LA can also induce apoptosis of colon cancer cells through a reactive oxygen species- (ROS-) mediated endoplasmic reticulum (ER) stress pathway [9]. He et al. administered LA orally to rats and measured some major pharmacokinetic parameters; the rate of elimination is $0.183 \pm 0.02 \mathrm{~h}^{-1}$, the rate of absorption is $0.781 \pm 0.08 \mathrm{~h}^{-1}$, the rate of distribution is $0.815 \pm 0.06 \mathrm{~h}^{-1}, t_{1 / 2 \beta}$ is $3.789 \pm 0.38 \mathrm{~h}, t_{1 / 2 \mathrm{a}}$ is $0.857 \pm 0.09 \mathrm{~h}$, $T_{\max }$ is $0.5 \pm 0.05 \mathrm{~h}$, and $C_{\max }$ is $1149.2 \pm 103.43 \mathrm{ng} / \mathrm{mL}$ [10]. 


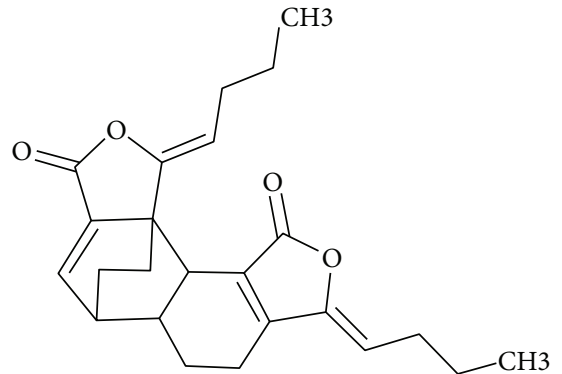

(a)

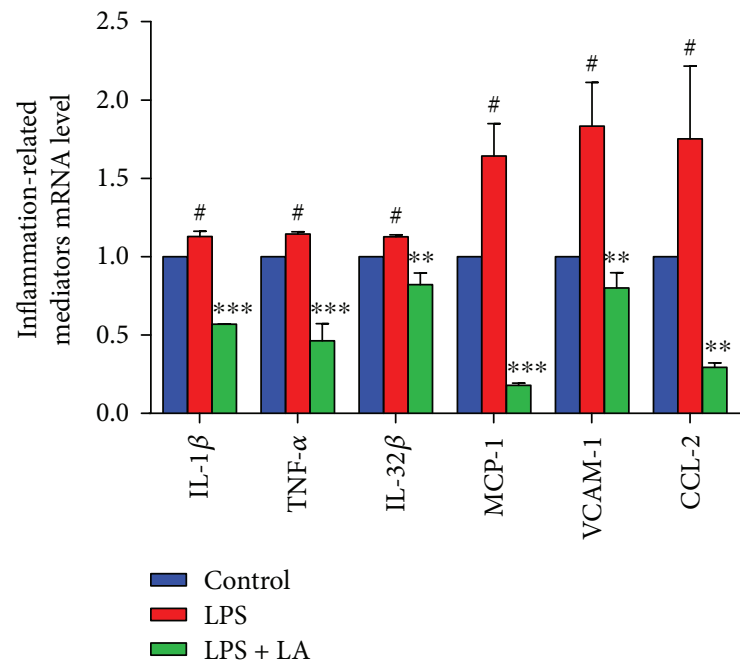

(b)
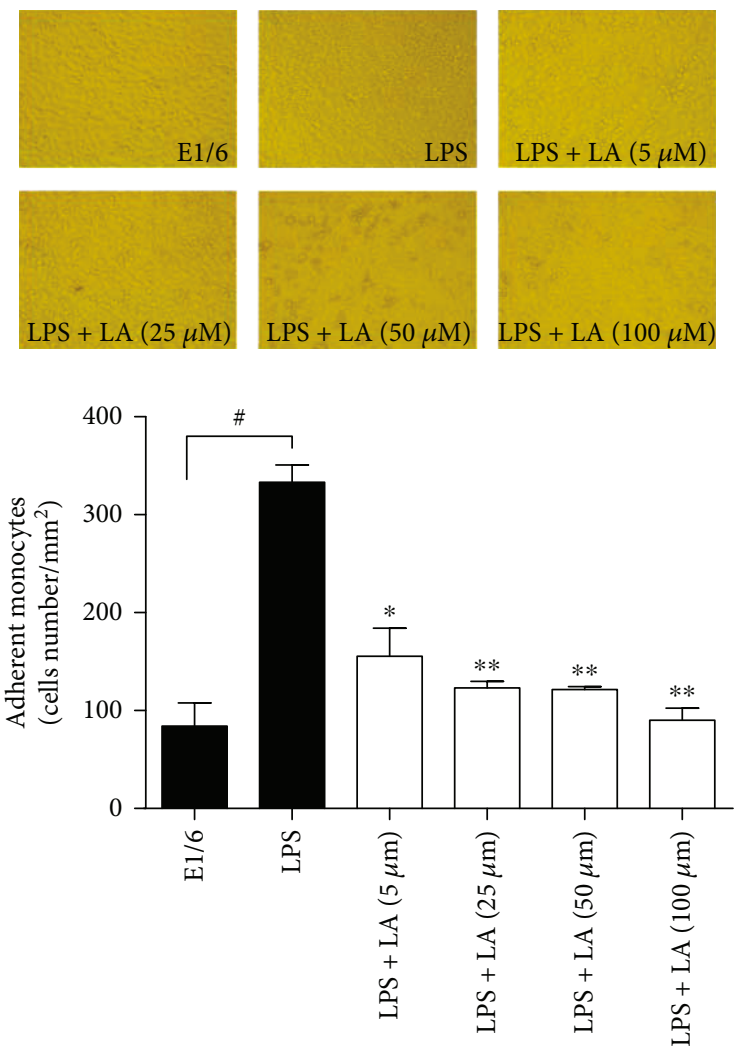

(c)

FIGURE 1: LA inhibited the mRNA levels of LPS-induced proinflammatory mediators and inhibited the adhesion of monocytes to HUVECs. The molecular structure of LA (a). HUVECs were pretreated with $50 \mu \mathrm{M}$ of LA for $60 \mathrm{~min}$ before treatment with LPS (100 ng/mL) (b) for $6 \mathrm{~h}$. Expression of IL- $\beta$, TNF- $\alpha$, IL-32 $\beta$, MCP-1, CCL-2, and VCAM-1 mRNA levels were measured using RT-PCR. HUVECs were pretreated with a blocking mouse antihuman monoclonal antibody (E1/6) against VCAM-1 or various doses of LA for 60 min before treatment with LPS. U937 mononuclear cells adhered to LPS-stimulated HUVECs were observed through microscopic images (c). Data represent the mean \pm SD of at least three independent experiments, and each experiment was performed in triplicate. ${ }^{\#} p<0.05$ versus control; ${ }^{*} p<0.05$ versus LPS; ${ }^{* *} p<0.01$ versus LPS; ${ }^{* * *} p<0.001$ versus LPS.

Xiong et al. proved that LA is an anti-inflammatory quality marker of Xiaojin pills [11]; Xiong et al.'s paper may be the only report describing LA's anti-inflammatory effects. However, the research about LA's anti-inflammatory biological mechanism has not yet been reported. Choi et al. found that ligustilide can attenuate vascular inflammation [12]. LA is a dimer of ligustilide; one can speculate that some connection may exist between LA and vasculitis. The purpose of this study was to explore the anti-inflammatory effects of LA, its biological mechanisms, and the effect of LA on TAO.

\section{Materials and Methods}

The experiment was divided into two parts, an in vitro experiment on human umbilical vein endothelial cells (HUVECs) and an in vivo experiment on rats.

2.1. Materials. All chemicals were purchased from commercial sources. HUVECs (CRL-1730) were purchased from ATCC (Manassas, VA, USA). LA (pure standard) was purchased from Shanghai Daoyi Biotechnology Co., Ltd. (Shanghai, China). Monoclonal mouse anti-GAPDH (glyceraldehyde 3-phosphate dehydrogenase) and horseradish peroxidase- (HRP-) conjugated antibody (KC-5G5) were purchased from KangChen Bio-tech Inc. (Shanghai, China). p-Syk (Tyr535/526), ERK (4695S), p-ERK (4370S), p38 (8690S), p-p38 (4511S), JNK (9258S), p-JNK (4668S), and IL-1 $\beta$ (12703S) antibodies were purchased from Cell Signaling Technology (Danvers, MA, USA). Antihuman pro-IL-18 (M156-3) and IL-18 (D043-3) antibodies were purchased from Medical \& Biological Laboratories (Nagoya, Japan). A Syk enzyme system (V3801) and ADP-Glo kinase assay (V9101) were purchased from Promega (Madison, WI, USA). Human pro-IL-1 $\beta$ (MAB6964-SP) antibody was purchased from R\&D Systems (Minneapolis, MN, USA). A high-capacity cDNA reverse transcription kit, Lipofectamine 2000, and TRIzol Reagent were purchased from Life Technologies (Carlsbad, CA, USA). An enhanced chemiluminescent (ECL) Immobilon Western Chemiluminescent HRP Substrate (WBKLS0500) was purchased from Millipore (Billerica, MAS, USA). JNK inhibitor (SP600125) and p38 
inhibitor (SB203580) were purchased from Selleck Chemicals (Houston, TX, USA).

Sodium laurate was purchased from Sinopharm Chemical Reagent Co., Ltd. (product number: 30168127; Shanghai, China). Medical glues were purchased from Guangzhou Baiyun Medical Adhesive Co., Ltd. (Guangzhou, China).

\subsection{In Vitro Experiments}

2.2.1. Cell Culture. HUVEC and HKE293 cells were maintained at $37^{\circ} \mathrm{C}$ in $5 \% \mathrm{CO}_{2}$ in Dulbecco's modified Eagle's medium containing $10 \%$ fetal bovine serum. The medium was changed every 2 days, and the cells were passaged with trypsin-EDTA. Cells at passages 4-8 were used for the studies.

2.2.2. Monocyte Cell Adhesion Assays. Monocyte U937 cells were grown in Roswell Park Memorial Institute 1640 medium containing $10 \%$ fetal calf serum. For the adhesion assays, HUVECs were grown to confluence in 6-well tissue culture plates, after which $100 \mathrm{ng} / \mathrm{mL}$ of lipopolysaccharide (LPS) was added for an additional $16 \mathrm{~h}$ to induce the expression of VCAM- 1 , in the presence or absence of LA $(0,5,25$, 50 , and $100 \mu \mathrm{mol} / \mathrm{L})$. For the control, monolayer cells were treated with a mouse antihuman monoclonal antibody (E1/6) against VCAM-1. Adhesion assays were performed by adding $1 \mathrm{~mL}$ of the concentrated U937 cells (with a density of $10^{6}$ cells $/ \mathrm{mL}$ ) to each monolayer under rotating conditions $(63 \mathrm{rpm})$ at $21^{\circ} \mathrm{C}$ and incubating for $10 \mathrm{~min}$. After nonadherent cells were removed by washing them gently three times with phosphate-buffered saline, the adherent cells in 6-well plates were fixed with $1 \%$ paraformaldehyde. The number of adherent cells was determined through counting six different fields by using an ocular grid and a 20x objective. Fields for counting adherent U937 cells were randomly selected at a half-radius distance from the center of the monolayers. Counts were performed in three independent experiments.

2.2.3. In Vitro Syk Activity Assay. Syk activity was measured according to the manufacturer's protocol (Promega, USA). Briefly, Syk $(100 \mathrm{ng} / \mu \mathrm{L})$, a substrate $(0.2 \mu \mathrm{g} / \mu \mathrm{L})$, adenosine triphosphate (ATP) $(10 \mu \mathrm{mol} / \mathrm{L})$, and LA $(0.05-$ $50,000 \mathrm{nmol} / \mathrm{L})$ were diluted in kinase buffer, and $5 \mu \mathrm{L}$ of inhibitors, $10 \mu \mathrm{L}$ of an enzyme, and $10 \mu \mathrm{L}$ of the substrate/ ATP mixture were added to each well of a 96-well plate. After incubation at room temperature for $60 \mathrm{~min}, 25 \mu \mathrm{L}$ of adenosine diphosphate- (ADP-) Glo $^{\mathrm{TM}}$ reagent was added to each well, followed by incubation at room temperature for $40 \mathrm{~min}$. Finally, $10 \mu \mathrm{L}$ of kinase detection reagent was added to each well, followed by incubation at room temperature for $30 \mathrm{~min}$. Chemiluminescent signal (integration time: $1 \mathrm{~s}$ ) was recorded using a Varioskan Flash microplate spectrophotometer (Thermo Scientific, USA).

2.2.4. RNA Isolation and Quantitative Polymerase Chain Reaction. Total RNA was extracted from the cells with Invitrogen TRIzol Reagent (Life Technologies) according to the manufacturer's protocol. First-strand complementary DNAs (cDNAs) were synthesized using a high-capacity
TABLE 1: Quantitative RT-PCR primer sequences.

\begin{tabular}{lcc}
\hline Gene & Direction & Primer \\
\hline TNF- $\alpha$ & Forward & CAGAGGGAAGAGTTCCCCAG \\
& Reverse & CCTTGGTCTGGTAGGAGACG \\
IL-1 $\beta$ & Forward & AAACAGATGAAGTGCTCCTTCCAGG \\
& Reverse & TGGAGAACACCACTTGTTGCTCCA \\
VCAM-1 & Forward & TTGCTCAGATTGGTGACTCCGTCT \\
& Reverse & TTCGTCACCTTCCCATTCAGTGGA \\
NLRP3 & Forward & TTCGGAGATTGTGGTTGGG \\
& Reverse & CAGGTAAAGGTGCGTGAGATT \\
IL-32 $\beta$ & Forward & GAGTTTCTGCTGCTCTCTGTCA \\
& Reverse & ATTTTGAGGATTGGGGTTCAG \\
eNOS & Forward & TCACCGCTACAACATCCT \\
& Reverse & CCTTCTGCTCATTCTCCA \\
GAPDH & Forward & AGAAGGCTGGGGCTCATTTG \\
NLRP3 & Feverse & AGGGGCCATCCACAGTCTTC \\
(rat) & Reverse & TGAAGAGTGTGATCTGCGGAAAC \\
GAPDH & Forward & CAAGTCATGTGGCTGAAGCTGT \\
(rat) & Reverse & ACGCCAGTAGACTCCACGACAT \\
\hline
\end{tabular}

cDNA reverse transcription kit (Life Technologies). The obtained cDNA was mixed with Maxima SYBR Green quantitative polymerase chain reaction (qPCR) Master Mix (Life Technologies) and gene-specific primers (Shanghai Generay Biotech, China). The sequences of the primers are shown in Table 1. QPCR was performed using a 7500 Fast Real-Time PCR System (Applied Biosystems, USA) according to the manufacturer's instructions. The amplification conditions consisted of an initial $15 \mathrm{~min}$ denaturation step at $95^{\circ} \mathrm{C}$, followed by 40 cycles of denaturation at $95^{\circ} \mathrm{C}$ for $15 \mathrm{~s}$, annealing at $55^{\circ} \mathrm{C}$ for $30 \mathrm{~s}$, and elongation at $72^{\circ} \mathrm{C}$ for $30 \mathrm{~s}$. The dissociation curves were analyzed to ensure the amplification of a single PCR product. Three independent assays were performed for each primer. The amount of cDNA was calculated for each sample from the standard curve. The relative expression was shown after normalization by the gene expression of GAPDH.

2.2.5. Western Blot. The cells were lysed in ice-cold radioimmunoprecipitation assay buffer $(50 \mathrm{mmol} / \mathrm{L}$ Tris/ $\mathrm{HCl}$, $\mathrm{pH} 8.0,150 \mathrm{mmol} / \mathrm{L} \mathrm{NaCl}, 2 \mathrm{mmol} / \mathrm{L}$ sodium orthovanadate, $1 \%$ Nonidet P-40, $1 \%$ sodium deoxycholate, $0.1 \%$ sodium dodecyl sulfate, $0.1 \mathrm{mmol} / \mathrm{L}$ dithiothreitol, $0.05 \mathrm{mmol} / \mathrm{L}$ phenylmethylsulfonyl fluoride, $0.002 \mathrm{mg} / \mathrm{mL}$ aprotimin, and $0.002 \mathrm{mg} / \mathrm{mL}$ leupeptin) and sonicated using a JY92-2D ultrasonic homogenizer (Ningbo Scientz Biotechnology, Zhejiang, China). Lysates were precleared through centrifugation at $12000 \times \mathrm{g}$ for $10 \mathrm{~min}$ at $4^{\circ} \mathrm{C}$. Aliquots of the cell lysates (50 or $100 \mu \mathrm{g}$ of each sample) were resolved on sodium dodecyl sulfate polyacrylamide gel electrophoresis and blotted onto nitrocellulose membranes (Pall China, Shanghai, China). The membrane was blocked in 5\% skim milk overnight at $4^{\circ} \mathrm{C}$. This was followed by incubation with primary antibodies for $2 \mathrm{~h}$ and exposure to a horseradish 
peroxidase-conjugated secondary antibody for $1 \mathrm{~h}$ at room temperature. Visualization was performed using ECL Immobilon Western Chemiluminescent HRP Substrate (Millipore). The membrane was exposed to a highperformance autoradiographic film, (Fuji Film, Tokyo, Japan) and visualized using the ECL Immobilon Western Chemiluminescent HRP Substrate (WBKLS0500; Millipore). A quantitative analysis was performed using Quantity One software. Western blot experiments were performed at least three times.

\subsection{In Vivo Experiment}

2.3.1. Animal Ethics Statement and Animals. The animals received care in compliance with the Guide for the Care and Use of Laboratory Animals published by the US National Institutes of Health. The animal experiments in this study were approved by the Animal Ethics Committee of Shanghai University of Traditional Chinese Medicine (approval number SZY201602004).

Male Wistar rats were purchased from Vital River Experimental Animal Technology Co., Ltd. (Beijing, China). Rats were housed in cages (three per cage) maintained at constant humidity $(65 \% \pm 5 \%)$ and temperature $\left(24^{\circ} \mathrm{C} \pm 1^{\circ} \mathrm{C}\right)$ with a 12-hour light-dark cycle. Rats were allowed ad libitum access to tap water and food throughout the experimental protocols. Thirty-six male Wistar rats were randomly divided into four groups (with nine rats in each): the control group, positive control group (inject of sodium laurate into the femoral artery of the lower extremity to make TAO model), negative control group (inject of saline solution into the femoral artery of the lower extremity), and TAO and LA group. Rats in the TAO and LA group were treated with LA for 16 days $(20 \mathrm{mg} / \mathrm{kg} /$ day, 2 days before surgery, and 2 weeks after surgery). By contrast, rats in the control group, positive control group, and negative control group were given the adjuvant $(0.30 \% \mathrm{CMC}-\mathrm{Na})$ only. Two weeks after surgery, rats were anesthetized using an intraperitoneal injection of sodium pentobarbital at a dose of $45 \mathrm{mg} / \mathrm{kg}$. Blood was taken from the abdominal aorta, and serum was separated; the right lower limb aorta was isolated and then stored at $-80^{\circ} \mathrm{C}$.

2.3.2. Rat TAO Model. Before surgery, the baseline blood flow was measured through laser speckle flowmetry with the PeriCam PSI System (Perimed AB, Stockholm, Sweden). An intraperitoneal injection of $3 \%$ sodium pentobarbital $(45 \mathrm{mg} / \mathrm{kg})$ was administered to anesthetize each rat; each injected rat was then fixed on a plate. The right groin was taken as the surgical position, which was shaved and disinfected with iodophor. The midpoint of the groin was incised approximately $1.5 \mathrm{~cm}$, and the subcutaneous tissue and muscle tissue were bluntly dissected, exposing the femoral artery. Then an artery clip was used to block the blood flow of the proximal heart end to separate the superficial part of the abdominal artery (approximately $0.5 \mathrm{~cm}$ in length), and $10 \mathrm{mg} / \mathrm{mL}$ sodium laurate $0.2 \mathrm{~mL}$ was injected to the distal direction [13-15]; further, medical glue was used to close the puncture point. Next, the artery clip was opened $1 \mathrm{~min}$ after surgery, and after confirming that there was no bleeding, sutures were applied to the skin. The same surgical method was used for the negative control group, but $0.2 \mathrm{~mL}$ of physiological saline was injected instead of sodium laurate. After the operation, the rats were placed on a thermal pad for $10 \mathrm{~min}$ to recover body temperature. The effect of the operation was confirmed using laser speckle flowmetry $30 \mathrm{~min}$ after surgery. The blood flow was then monitored at 2, 5, 8, 11 , and 14 days.

2.3.3. Enzyme-Linked Immunosorbent Assay. An enzymelinked immunosorbent assay (ELISA) was performed using a rat IL- $1 \beta$ ELISA kit (RLB00, R\&D Systems, USA), rat interleukin-6 (ERC003.96, Xinbosheng Biological Technology Co., Ltd., Shenzhen, China), and C-reactive protein (CRP) ELISA assay kits (H126, Nanjing Jiancheng Bioengineering Institute, Nanjing, China); all kits were used according to the manufacturers' instructions.

2.4. Statistical Analysis. Data were analyzed using GraphPad Prism 5 or SPSS 15.0 software. A paired $t$-test was used to compare the two groups, and a one-way analysis of variance was used for comparisons within groups. Data are expressed as mean \pm standard deviation (SD), and $p<0.05$ was considered significant.

\section{Results}

3.1. In Vitro Results. To test the anti-inflammatory effects of LA, real-time qPCR was employed to detect the messenger RNA (mRNA) expression of different proinflammatory genes. After the HUVECs were stimulated using LPS for $6 \mathrm{~h}$, the mRNA expressions of IL- $1 \beta$, TNF- $\alpha$, IL-32 $\beta$, MCP1 , CCL-2, and VCAM-1 were increased by 1.13 - to 1.83 -fold compared to control groups. However, pretreatment with LA ( $50 \mu \mathrm{mol} / \mathrm{L})$ for 1 hour prior to LPS stimulation almost completely blocked the enhanced mRNA expressions near or below the baseline (Figure 1(b)). The effects of LA on monocyte adhesion to endothelial cells mediated by VCAM-1 were examined. As presented in Figure 1(c), after incubation of the HUVECs with LPS (16 h), adherent U937 monocytes markedly increased to $74.0 \pm 3.9$ cells $/ \mathrm{mm}^{2}$, which was almost completely blocked by treatment with a mouse antihuman monoclonal E1/6 antibody against VCAM-1. Pretreatment with various doses of LA (5$100 \mu \mathrm{mol} / \mathrm{L})$ inhibited U937 monocyte adhesion in a dosedependent manner. Monocyte adhesion to the HUVECs significantly decreased after LA treatment from $34.5 \pm 6.4$ cells $/ \mathrm{mm}^{2}(5 \mu \mathrm{mol} / \mathrm{L}, \quad p<0.05)$ to $20.0 \pm 2.8$ cells $/ \mathrm{mm}^{2}$ $(100 \mu \mathrm{mol} / \mathrm{L}, p<0.005)$.

Next, the effects of LA on Syk activity were examined. The results revealed that LPS induced Syk phosphorylation and nonphosphorylated Syk depletion, whereas increased doses of LA inhibited Syk phosphorylation (Figure 2(a)). In order to confirm whether LA mediated the mitogenactivated protein kinase (MAPK) pathway, activation of ERK, p38, and JNK was examined by Western blotting. The results show that LA inhibited the activation of p38 and JNK in LPS-stimulated HUVECs. Compare with the LPS group, phosphorylation levels of p38 and JNK in LA 

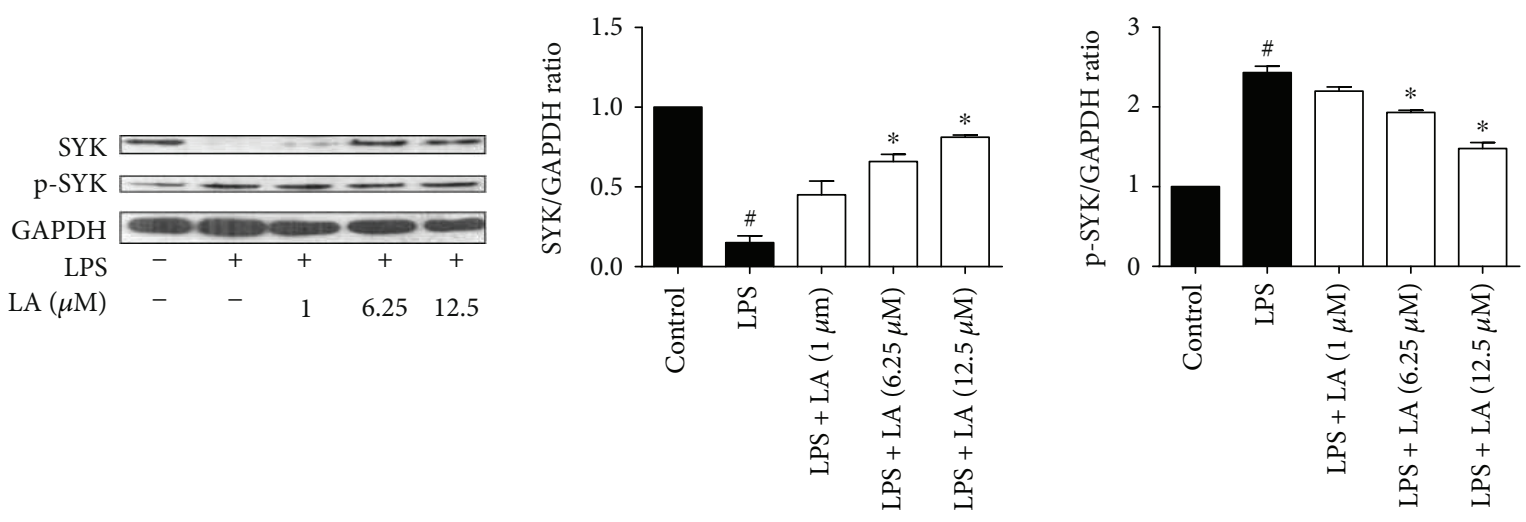

(a)
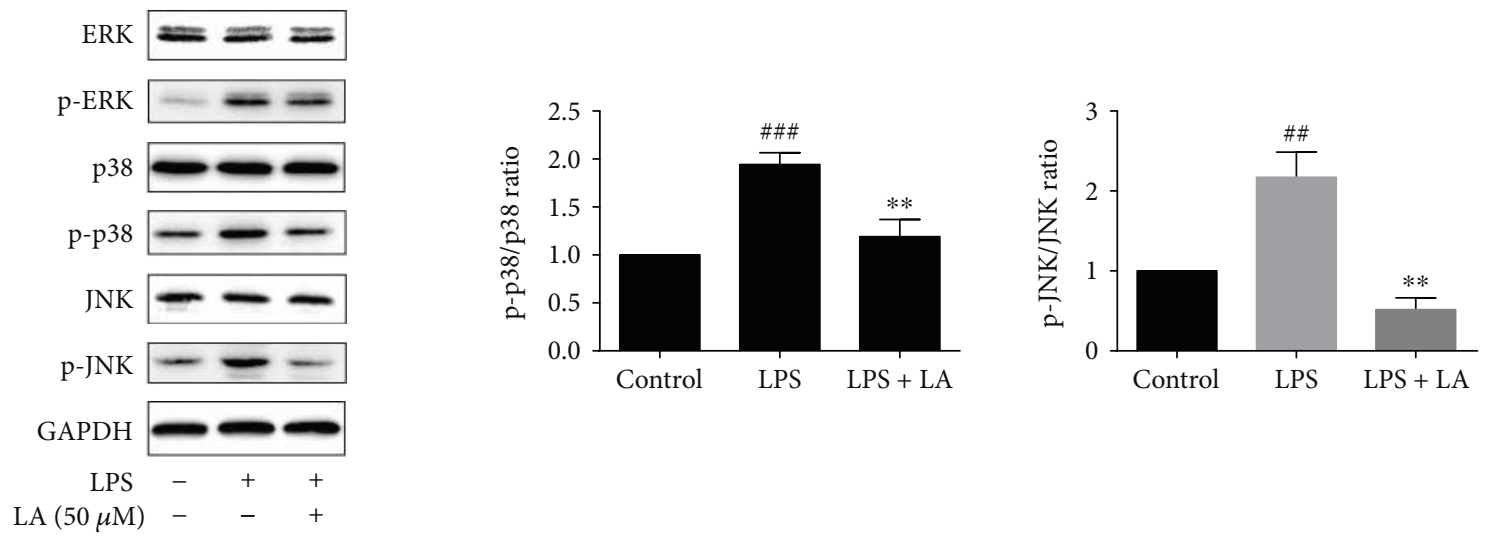

(b)

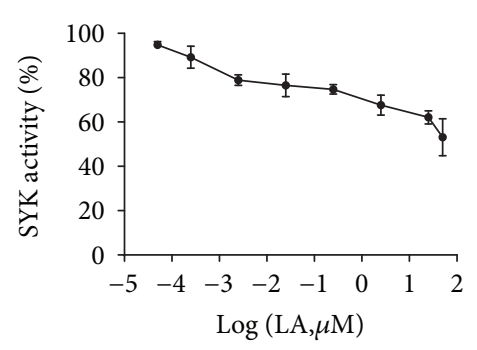

(c)

FIGURE 2: LA inhibited Syk and p38/JNK activity. HUVECs were pretreated in the presence of various doses of LA for $1 \mathrm{~h}$ and stimulated with LPS for $30 \mathrm{~min}$. A Western blotting analysis was performed to evaluate the levels of phosphorylated Syk and total Syk (a). HUVECs were treated with $50 \mu \mathrm{M}$ of LA for $1 \mathrm{~h}$ before LPS stimulation for $15 \mathrm{~min}$, and then the total proteins of cells were extracted. The activation of the MAPK (b) pathways was detected using Western blotting with specific antibodies. Syk activity was analyzed using an in vitro kinase assay through recombinant Syk obtained from Promega. The assay (ADP-Glo kinase assay) was performed to measure luminescent signals by using Ultra-Glo Luciferase; its ADP from a kinase reaction is converted into ATP converted into light. Kinase assays were conducted in the presence of various doses of LA for $30 \mathrm{~min}$. The results were evaluated using Syk activity (c). Data represent the mean \pm $\mathrm{SD}$ of at least three independent experiments, and each experiment was performed in triplicate. ${ }^{\#} p<0.05{ }^{\# \#} p<0.01$ and ${ }^{\# \# \#} p<0.001$ versus control alone; ${ }^{*} p<0.05^{* *} p<0.01$ versus LPS alone.

pretreated cells were reduced by 1.74 and 4.36 times, respectively (Figure 2(b)). To test whether LA directly blocked Syk activity, Syk kinase assay kit was used to determine the inhibitory effects of LA; the results revealed that LA inhibited Syk activity in a dose-dependent manner with an EC50 value $0.129 \mu \mathrm{mol} / \mathrm{L}$ (Figure 2(c)).

To observe the effect of LA on NLRP3 (a downstream gene of Syk), the expression of NLRP3 was detected through RT-PCR. After HUVEC cells were stimulated by LPS for $6 \mathrm{~h}$, the mRNA expressions of NLRP3 was increased by 1.33 -fold compared to the control group. However, pretreatment with LA for $1 \mathrm{~h}$ prior to LPS stimulation the expression of NLRP3 is 0.84 times lower than control group. The results indicated that LA could downregulate NLRP3 gene expression (Figure 3(a)). The NLRP3 inflammasome was responsible for the expression of mature IL-1 $\beta$ and IL-18. Western blotting was used to elucidate the role of LA in LPSinduced IL-1 $\beta$ and IL-18 release. The results showed that 


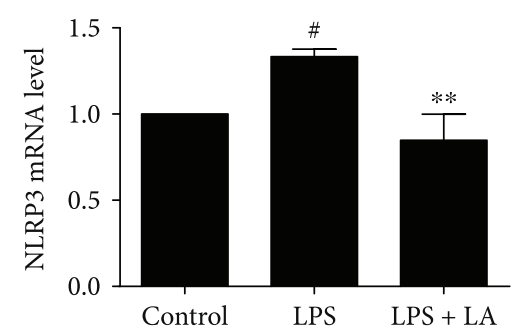

(a)
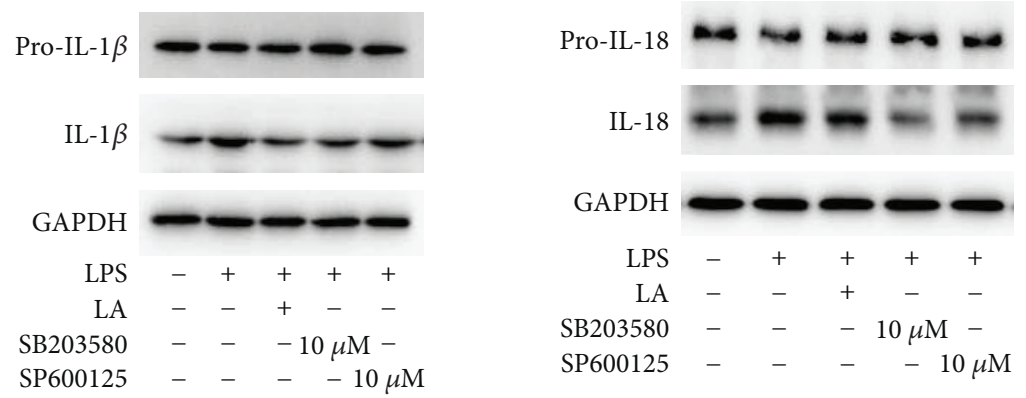

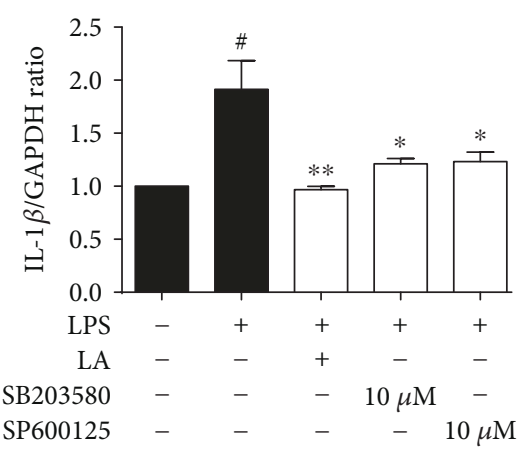

(b)

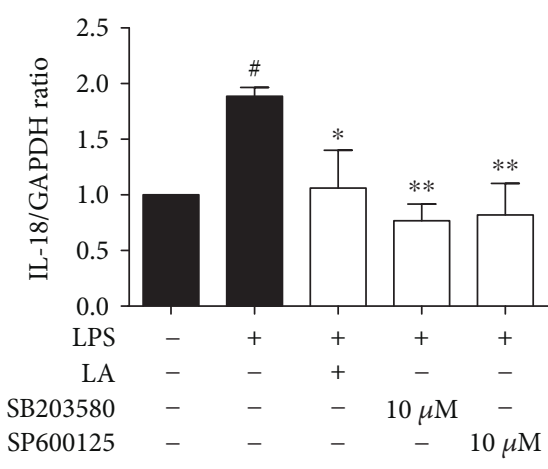

(c)

FIGURE 3: LA suppressed NLRP3 gene and downstream related protein expression. HUVECs were treated with $50 \mu \mathrm{M}$ of LA for $1 \mathrm{~h}$, and then LPS was stimulated for $24 \mathrm{~h}$. The NLRP3 mRNA level (a) was analyzed using qRT-PCR. LA and p38/JNK inhibitors (SB203580 and SP600125) were treated with HUVECs for $60 \mathrm{~min}$ before LPS stimulation for $24 \mathrm{~h}$. NLRP3-associated proteins IL- $1 \beta$ and IL-18 were detected using immunoblotting $(\mathrm{b})(\mathrm{c})$. Data represent the mean \pm SD of at least three independent experiments, and each experiment was performed in triplicate. ${ }^{\#} p<0.05$ versus control; ${ }^{*} p<0.05{ }^{* *} p<0.01$ versus LPS.

LA substantially blocked the LPS-induced upregulation of IL- $1 \beta$ and IL-18 compared with treatment with LPS alone. This effect was similar to the JNK and p38 inhibitors (Figures 3(b) and 3(c)). These results indicated that LA might reduce the promotion of the NLRP3 inflammasome to inhibit inflammation.

3.2. In Vivo Results. After injection with sodium laurate, different degrees of ischemic necrosis occurred in the right lower limbs of rats in the positive control group and the $\mathrm{TAO}+\mathrm{LA}$ group (Figure 4(a)). According to the vasculitis model score criteria [16], the score of the positive control group was significantly higher than that of the TAO + LA group after surgery for 2 weeks (Figure 4(b)). Then, laser speckle flowmetry was used to determine the blood flow of the lower extremities of the rats in each group. The blood flow of the negative control group was higher than that of the positive control group and $\mathrm{TAO}+\mathrm{LA}$ group after the operation for $30 \mathrm{~min}$. The $\mathrm{TAO}+\mathrm{LA}$ group was treated intragastrically 2 days before surgery; the blood flow of the $\mathrm{TAO}+\mathrm{LA}$ group was higher than that of the positive control group. The blood flow in the right legs of the negative control group returned to normal 2 weeks after the operation, whereas the blood flow in the right legs of the TAO + LA group and positive control group was lower than that of the control group, and the blood flow intensity in the $\mathrm{TAO}+\mathrm{LA}$ group was higher than that in the positive control group (Figure 4(c)). Two weeks after the operation, the right lower extremity muscles of each group were examined using hematoxylin and eosin (HE) staining. The degree of muscular inflammatory necrosis in the positive control group was significantly higher than in the TAO + LA group (Figure $4(\mathrm{~d})$ ).

The levels of inflammatory cytokines were detected through ELISA. The serum IL- $1 \beta$, IL-6, and CRP in the positive control group was higher than those in the control 


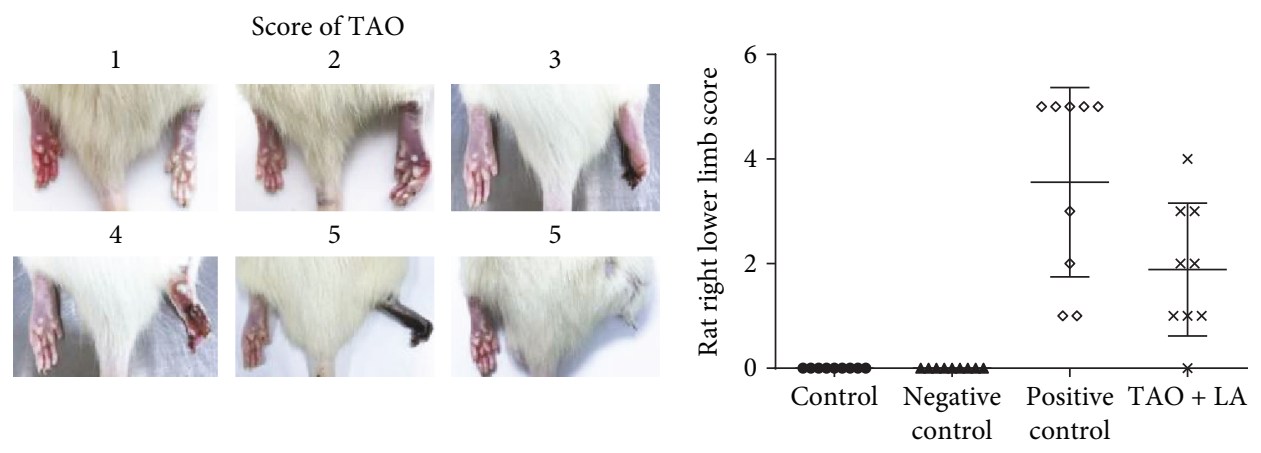

(a)

(b)
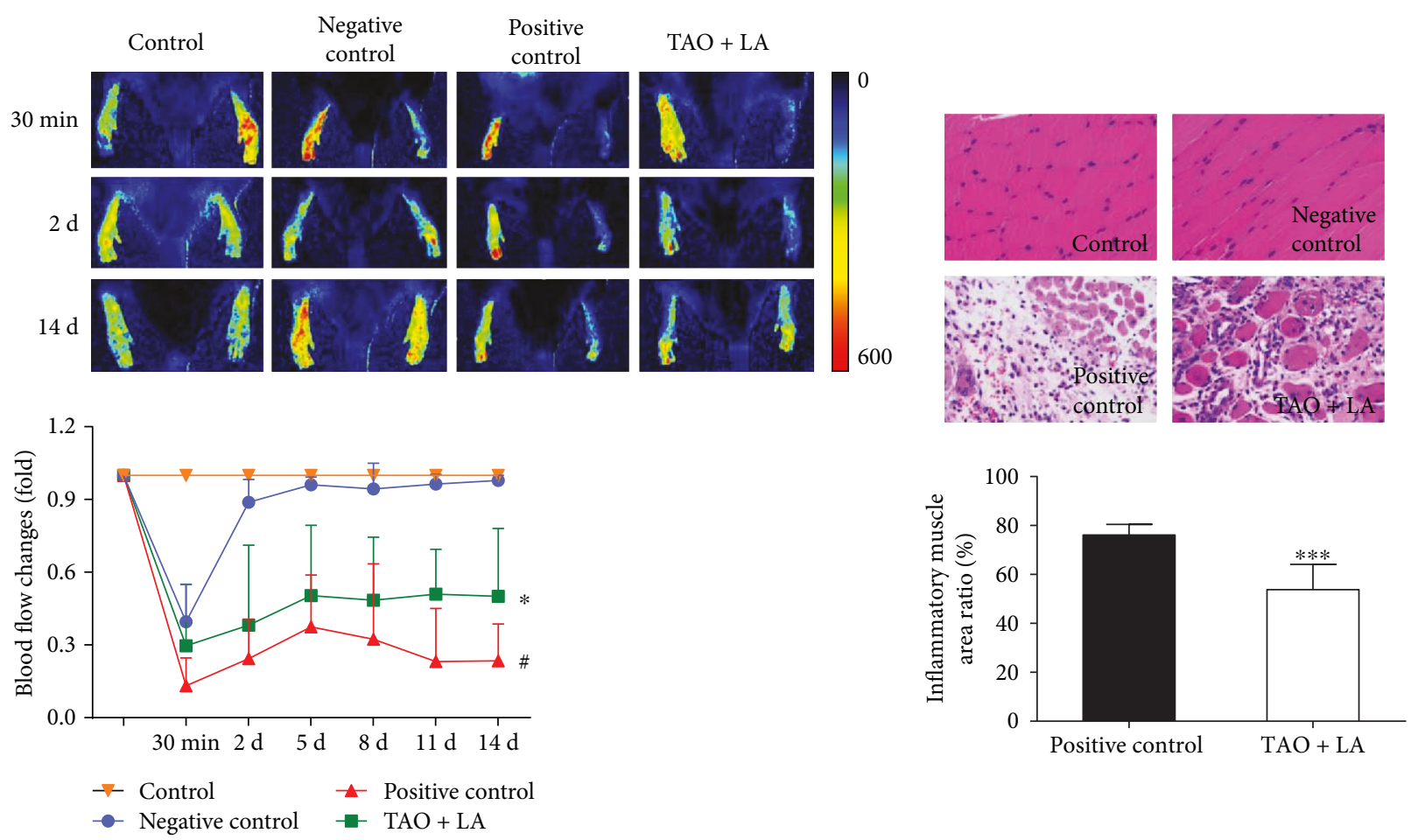

(c)

(d)

FIgure 4: LA reduced the damage to the rats' lower limbs in the TAO model. A right lower extremity arterial injection of sodium laurate $10 \mathrm{mg} / \mathrm{mL} 0.2 \mathrm{~mL}$ was used in the positive control group, and $0.9 \%$ physiological saline $0.2 \mathrm{~mL}$ femoral artery injection was used in the negative control group. The effect of the operation was confirmed using laser speckle flowmetry 30 min after surgery. The rats in the control group and positive and negative control groups were given the adjuvant (CMC-Na) only, whereas the LA group received $20 \mathrm{mg} / \mathrm{kg} / \mathrm{d}$ for 2 weeks, which started to gavage $2 \mathrm{~d}$ before surgery. One week after surgery, various degrees of gangrene were observed in the rats' lower right limbs, as shown in (a). The score of the right lower limb of each group was assessed according to the TAO model score standard (b). The blood flow of the lower limbs in each group was measured using laser speckle flowmetry (c). Inflammatory necrosis of the right leg muscle was observed using HE-stained slices (400*) (d). Data are presented as mean \pm SD. ${ }^{\#} p<0.05$ versus negative control group; ${ }^{*} p<0.05$ and ${ }^{* * *} p<0.001$ versus positive control group.

group and negative control group. Compared with the positive control group, the serum IL-1 $\beta$, IL-6, and CRP levels were significantly decreased in the TAO + LA group rats (Figures $5(\mathrm{a})-5(\mathrm{c}))$. LA reduced the expression of NLRP3 in the rats' lower limb arteries (Figure 5(d)).

\section{Discussion}

Liu et al. proposed that inflammation and the immune system play central roles in TAO pathogenesis [17]. LPS is a component of Gram-negative bacteria involved in the transcription of numerous proinflammatory genes. LPS is a commonly used stimulator of inflammation models and is often used for in vitro mechanism studies of TAO [18, 19]. Toll-like receptor 4 (TLR4) is activated by LPS. TLR4 can induce the production of proinflammatory mediators; this effect of TLR4 can be applied to eradicate bacteria. CD14 transfers LPS to the TLR4/MD-2 complex, which dimerizes and triggers the MyD88- and TRIF-dependent production of proinflammatory cytokines and type I interferons [20]. 


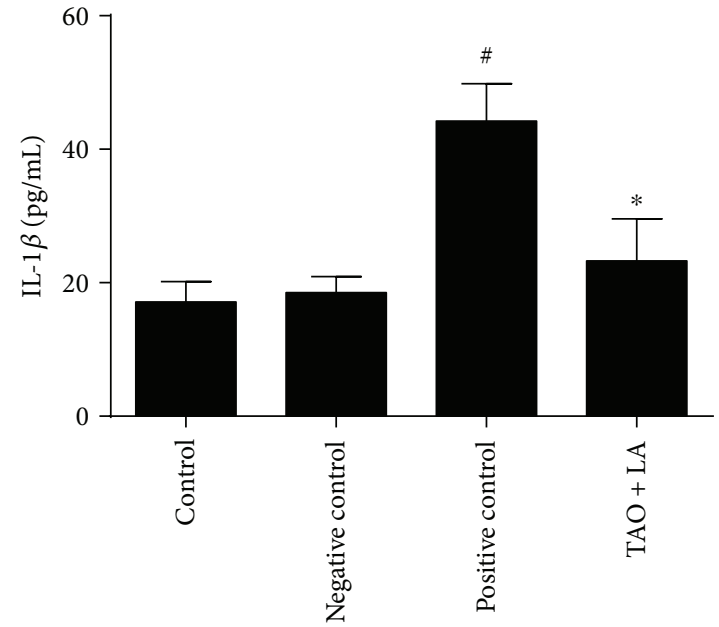

(a)

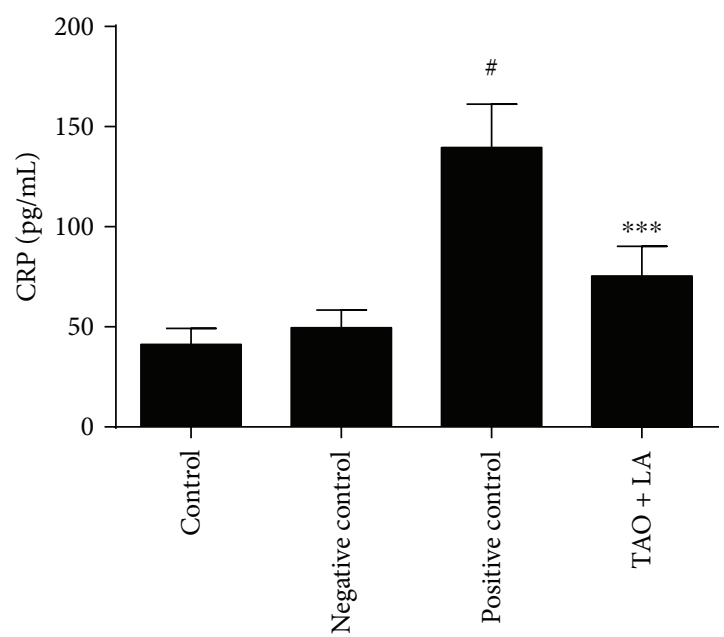

(c)

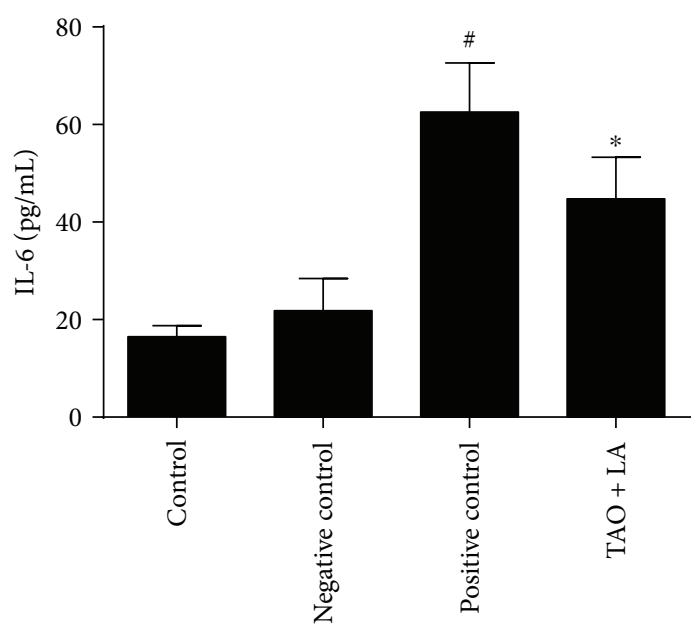

(b)

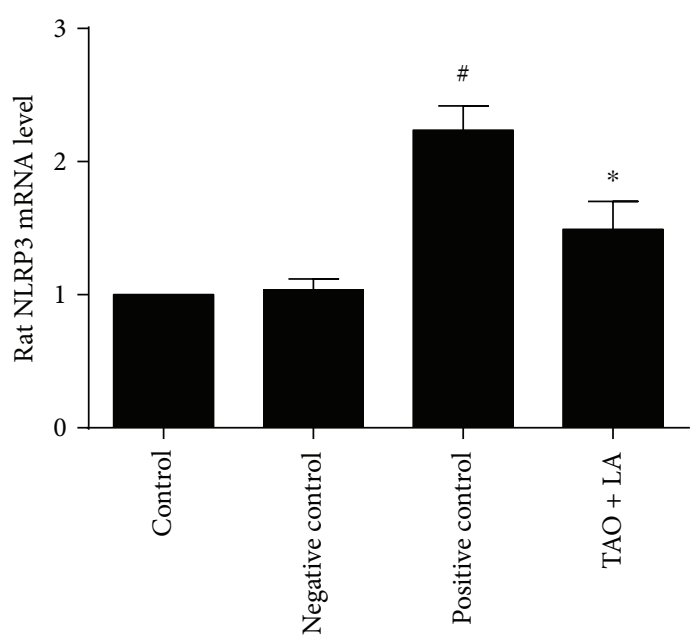

(d)

FIGURE 5: LA reduced the rats' inflammatory factor levels. Wistar rat serum IL-1 $\beta /$ IL-6/CRP content was detected using an ELISA (a-c). Lower limb arterial NLRP3 mRNA levels in rats (d). ${ }^{\#} p<0.05$ versus negative control group; ${ }^{*} p<0.05$ and ${ }^{* * *} p<0.001$ versus positive control group.

LPS-activated TRL4 activates the NFkappaB pathway and all three MAPK pathways (ERK, JNK/SAPK, and p38). LPS also stimulates NLRP3 inflammasomes, the bestcharacterized NLR molecules, which induces the release of mature IL-1 $\beta$ and IL-18 [21].

The study revealed that LA directly inhibited Syk activity and Syk phosphorylation. Syk plays a crucial role in adaptive immune receptor signaling and numerous biological processes, including cellular adhesion, innate immune recognition, osteoclast maturation, platelet activation, and vascular development [22]. Syk activity is induced by LPS, TNF- $\alpha$, IL-1, thrombin, C-response protein, minimally oxidized LDL, and high glucose levels [23-29]. It is transiently recruited to a complex containing MyD88, TLR4, and IRAK-1 upon LPS stimulation and disassociates over time [24]. Notably, the kinetics of TLR4 tyrosine phosphorylation coincides with an early wave of Syk tyrosine phosphorylation. Syk is also required for cytokine production with MyD88 adaptor protein pathways [30]. Numerous studies have demonstrated that Syk-mediated mitogen-activated protein kinases play a vital role in the expression of inflammatory genes [31-33]. Syk mediates NLRP3 inflammasome activation [34-36]. Therefore, one can infer that by inhibiting the phosphorylation of Syk, LA inhibits the activity of $\mathrm{p} 38 / \mathrm{JNK}$ and reduces both the expression of NLRP3 and the secretion of related proteins IL-1 $\beta$ and IL-18 (Figure 6).

TAO is a rare disease of unknown etiology, and despite its discovery more than a century ago, little progress has been made in determining its mechanism and suitable treatments. In a thrombohemorrhagic vasculitis model, the mouse vessel wall Syk tyrosine kinase signaling pathway was triggered. This led to neutrophil elastase release, which caused hemorrhage, fibrin deposition, and thrombosis [37]. Sun and colleagues proposed potential mechanisms from a genetic and immunoreactive perspective for its inception and revealed that the pathogenesis of TAO is due to a type of gene polymorphism that leads to 


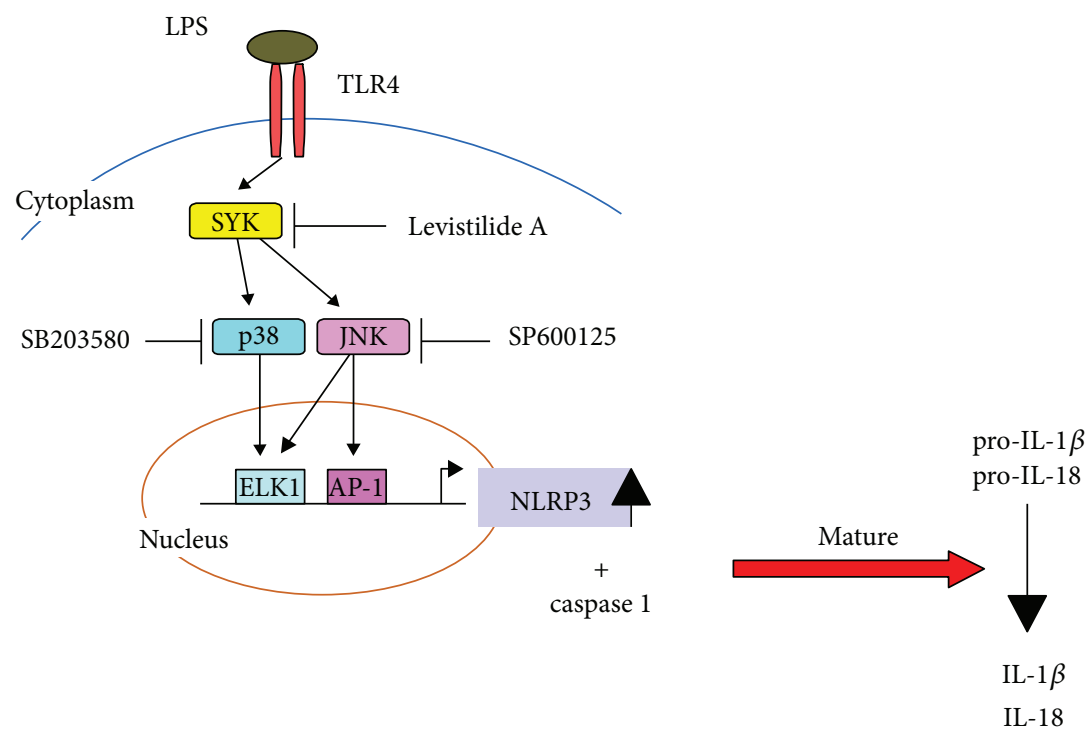

FIGURE 6: LA anti-inflammatory mechanism diagram. LA inhibited the activity of p38/JNK by decreasing Syk phosphorylation, thereby inhibiting the downstream NLRP3 inflammasome gene expression and the secretion of its related proteins (IL-1 $\beta$ and IL-18).

immunological inflammatory vasculitis, which is highly linked to HVECs and the TLR-MyD88-NFkB pathway [38]. Neutrophil aggregation and their interaction with the endothelium and platelets as well as neutrophil-derived microparticles promote the generation of occlusive thrombi, fibrin accumulation, and hemorrhage [39-41]. Monocyte aggregation contributes to thrombus formation. Studies have revealed that macrophages infiltrated the internal lamina and that VCAM-1 and ICAM-1 were expressed in the vascular endothelium of TAO lesions $[42,43]$. The endothelial cell experiment revealed that LA inhibited the adhesion of monocytes to endothelial cells. Animal studies have shown that LA reduces the degree of necrosis of the lower extremities in TAO rats, improves blood supply, and reduces the expression of NLRP3 in blood vessels. LA may achieve these benefits by reducing Syk activity and inflammatory damage to endothelial cells. This hypothesis has not yet been validated by in vivo experiments; this lack of confirmation may be one of the limits of the present study. Generalized functional arterial disorder in patients with $\mathrm{TAO}$ with functional deterioration of the brachial artery could be related to increased levels of various inflammatory markers [3]. The serum levels of IL- 6 and IL-8 significantly decreased after TAO was treated with conventional therapy [44]. This was consistent with the decreases in serum IL-6, IL-8, and CRP after treatment of TAO rats with LA.

Few pharmacological and surgical options are available to treat TAO other than TAO therapy in addition to the discontinuation of cigarette smoking [45]. The frequent use of high-potency vasodilators, such as iloprost, bosentan, sildenafil, or alprostadil, relieves symptoms and reduces the risk of amputation. Although two randomized trials have demonstrated the efficacy of intravenous iloprost, no oral drug has been demonstrated to be effective in treating TAO. This study provides a biological mechanism for the anti-inflammatory effects of LA and a theoretical basis for the development of a drug for treating TAO.

\section{Conclusions}

LA displayed anti-inflammatory properties; some potential mechanisms involved the Syk-p38/JNK signal pathway. The results of this study indicate that LA can treat vascular diseases associated with inflammation such as TAO.

\author{
Abbreviations \\ GAPDH: Glyceraldehyde 3-phosphate dehydrogenase \\ HEK293: Human embryonic kidney cells 293 \\ HRP: Horseradish peroxidase \\ HUVECs: Human umbilical vein endothelial cells \\ IL: Interleukin \\ LA: $\quad$ Levistilide A \\ LPS: Lipopolysaccharide \\ MCP-1: Monocyte chemotactic protein-1 \\ PBS: $\quad$ Phosphate-buffered saline \\ Syk: $\quad$ Spleen tyrosine kinase \\ U937: Human leukemic monocyte lymphoma cell line \\ VCAM: Vascular cell adhesion molecule \\ TAO: Thromboangiitis obliterans.
}

\section{Data Availability}

The data used to support the findings of this study are available from the corresponding author upon request.

\section{Conflicts of Interest}

The authors confirm that they have no conflicts of interest. 


\section{Authors' Contributions}

Huining Guo performed most experimental work and wrote the paper. Li Sun performed a part of the experiment. Shuang Ling performed the initial experiment and revealed the drug activity. Jin-Wen Xu conceived of the study.

\section{Acknowledgments}

This work was supported by grants from the National Science and Technology Major Project of the China Ministry of Science and Technology (2009ZX09311-003), the National Natural Science Foundation of China (81274130), the Innovation Program of Shanghai Municipal Education Commission of China (12ZZ1225), and the Shanghai 085 Project of Higher Education Connotation Construction (085ZY1202).

\section{References}

[1] N. Maugeri, P. Rovere-Querini, M. Baldini, M. G. Sabbadini, and A. A. Manfredi, "Translational mini-review series on immunology of vascular disease: mechanisms of vascular inflammation and remodelling in systemic vasculitis," Clinical \& Experimental Immunology, vol. 156, no. 3, pp. 395-404, 2009.

[2] K. Igari, T. Kudo, T. Toyofuku, and Y. Inoue, "Endothelial dysfunction in patients with Buerger disease," Vascular Health and Risk Management, vol. 13, pp. 317-323, 2017.

[3] M. Joras, P. Poredos, and Z. Fras, "Endothelial dysfunction in Buerger's disease and its relation to markers of inflammation," European Journal of Clinical Investigation, vol. 36, no. 6, pp. 376-382, 2006.

[4] S. L. Li, S. S. Chan, G. Lin et al., "Simultaneous analysis of seventeen chemical ingredients of Ligusticum chuanxiong by on-line high performance liquid chromatography-diode array detector-mass spectrometry," Planta Medica, vol. 69, no. 5, pp. 445-451, 2003.

[5] G. H. Lu, K. Chan, Y. Z. Liang et al., "Development of high-performance liquid chromatographic fingerprints for distinguishing Chinese Angelica from related umbelliferae herbs," Journal of Chromatography A, vol. 1073, no. 1-2, pp. 383-392, 2005.

[6] L. Lim, P. Shen, Y. Gong, and E. Yong, "Dimeric progestins from rhizomes of Ligusticum chuanxiong," Phytochemistry, vol. 67, no. 7, pp. 728-734, 2006.

[7] X. N. Li, Y. Y. Chen, D. P. Cheng, S. Q. Tong, H. B. Qu, and J. Z. Yan, "Two phthalide dimers from the radix of Angelica sinensis," Natural Product Research, vol. 26, no. 19, pp. 1782-1786, 2012.

[8] T. F. Lee, Y. L. Lin, and Y. T. Huang, "Studies on antiproliferative effects of phthalides from Ligusticum chuanxiong in hepatic stellate cells," Planta Medica, vol. 73, no. 6, pp. 527534, 2007.

[9] Y. Yang, Y. Zhang, L. Wang, and S. Lee, "Levistolide a induces apoptosis via ROS-mediated ER stress pathway in colon cancer cells," Cellular Physiology and Biochemistry, vol. 42, no. 3, pp. 929-938, 2017.

[10] W. Q. He, W. S. Lv, Y. Zhang et al., "Study on pharmacokinetics of three preparations from levistolide A by LC-MS-MS," Journal of Chromatographic Science, vol. 53, no. 8, pp. 12651273, 2015.
[11] X. Xiong, Y. N. He, B. Feng et al., "Screening for the anti-inflammation quality markers of Xiaojin Pills based on HPLC-MS/MS method, COX-2 inhibition test and protein interaction network," Scientific Reports, vol. 8, no. 1, p. 7454, 2018.

[12] E. S. Choi, J. J. Yoon, B. H. Han et al., "Ligustilide attenuates vascular inflammation and activates $\mathrm{Nrf} 2 / \mathrm{HO}-1$ induction and, NO synthesis in HUVECs," Phytomedicine, vol. 38, pp. 12-23, 2018.

[13] X. Kong, H. Yuan, X. Wu et al., "High-mobility-group box protein 1 A box reduces development of sodium laurateinduced thromboangiitis obliterans in rats," Journal of Vascular Surgery, vol. 57, no. 1, pp. 194-204, 2013.

[14] F. Hong, C. He, X. Liu, G. Tu, F. Guo, and S. Yang, "Protective effect of Shenfu injection on thromboangiitis obliterans model rats," Journal of Ethnopharmacology, vol. 138, no. 2, pp. 458462, 2011.

[15] Y. Xu, R. Zhang, J. Chen et al., "Urocortin promotes the development of vasculitis in a rat model of thromboangiitis obliterans via corticotrophin-releasing factor type 1 receptors," British Journal of Pharmacology, vol. 157, no. 8, pp. 13681379, 2009.

[16] J. Yu, E. D. deMuinck, Z. Zhuang et al., "Endothelial nitric oxide synthase is critical for ischemic remodeling, mural cell recruitment, and blood flow reserve," Proceedings of the National Academy of Sciences of the United States of America, vol. 102, no. 31, pp. 10999-11004, 2005.

[17] J. D. Martin-Rufino, F. S. Lozano, A. M. Redondo et al., "Sequential intravenous allogeneic mesenchymal stromal cells as a potential treatment for thromboangiitis obliterans (Buerger's disease)," Stem Cell Research \& Therapy, vol. 9, no. 1, p. $150,2018$.

[18] H. Liu, S. Ma, H. Xia, H. Lou, F. Zhu, and L. Sun, "Antiinflammatory activities and potential mechanisms of phenolic acids isolated from Salvia miltiorrhiza f. alba roots in THP-1 macrophages," Journal of Ethnopharmacology, vol. 222, pp. 201-207, 2018.

[19] E. S. Slavov, S. A. Stanilova, D. P. Petkov, and Z. G. Dobreva, "Cytokine production in thromboangiitis obliterans patients: new evidence for an immune-mediated inflammatory disorder," Clinical and Experimental Rheumatology, vol. 23, no. 2, pp. 219-226, 2005.

[20] A. Płóciennikowska, A. Hromada-Judycka, K. Borzęcka, and K. Kwiatkowska, "Co-operation of TLR4 and raft proteins in LPS-induced pro-inflammatory signaling," Cellular and Molecular Life Sciences, vol. 72, no. 3, pp. 557-581, 2015.

[21] S. Lacroix-Lamande, M. Fanton d'Andon, E. Michel et al., "Downregulation of the Na/K-ATPase pump by leptospiral glycolipoprotein activates the NLRP3 inflammasome," The Journal of Immunology, vol. 188, no. 6, pp. 2805-2814, 2012.

[22] A. Mócsai, J. Ruland, and V. L. J. Tybulewicz, "The SYK tyrosine kinase: a crucial player in diverse biological functions," Nature Reviews Immunology, vol. 10, no. 6, pp. 387-402, 2010.

[23] K. M. Bijli, F. Fazal, M. Minhajuddin, and A. Rahman, "Activation of Syk by protein kinase C- $\delta$ regulates thrombin-induced intercellular adhesion molecule- 1 expression in endothelial cells via tyrosine phosphorylation of RelA/p65," Journal of Biological Chemistry, vol. 283, no. 21, pp. 14674-14684, 2008.

[24] A. Chaudhary, T. M. Fresquez, and M. J. Naranjo, "Tyrosine kinase Syk associates with toll-like receptor 4 and regulates signaling in human monocytic cells," Immunology \& Cell Biology, vol. 85, no. 3, pp. 249-256, 2007. 
[25] Y. J. Kim, T. Y. Koo, W. S. Yang et al., "Activation of spleen tyrosine kinase is required for TNF- $\alpha$-induced endothelin-1 upregulation in human aortic endothelial cells," FEBS Letters, vol. 586, no. 6, pp. 818-826, 2012.

[26] Y. I. Miller, S.-H. Choi, P. Wiesner, and Y. S. Bae, “The SYK side of TLR4: signalling mechanisms in response to LPS and minimally oxidized LDL," British Journal of Pharmacology, vol. 167, no. 5, pp. 990-999, 2012.

[27] J. W. Xu, I. Morita, K. Ikeda, T. Miki, and Y. Yamori, "Creactive protein suppresses insulin signaling in endothelial cells: role of spleen tyrosine kinase," Molecular Endocrinology, vol. 21, no. 2, pp. 564-573, 2007.

[28] T. Yamada, S. Fujieda, S. Yanagi et al., "IL-1 induced chemokine production through the association of Syk with TNF receptor-associated factor-6 in nasal fibroblast lines," The Journal of Immunology, vol. 167, no. 1, pp. 283-288, 2001.

[29] W. S. Yang, J. W. Seo, N. J. Han et al., "High glucose-induced NF- $\kappa \mathrm{B}$ activation occurs via tyrosine phosphorylation of $\mathrm{I} \kappa \mathrm{B} \alpha$ in human glomerular endothelial cells: involvement of Syk tyrosine kinase," American Journal of Physiology-Renal Physiology, vol. 294, no. 5, pp. F1065-F1075, 2008.

[30] K. M. Dennehy, G. Ferwerda, I. Faro-Trindade et al., "Syk kinase is required for collaborative cytokine production induced through Dectin-1 and Toll-like receptors," European Journal of Immunology, vol. 38, no. 2, pp. 500-506, 2008.

[31] S. H. Choi, P. Wiesner, F. Almazan, J. Kim, and Y. I. Miller, "Spleen tyrosine kinase regulates AP-1 dependent transcriptional response to minimally oxidized LDL," PLoS One, vol. 7, no. 2, article e32378, 2012.

[32] P. G. Arndt, N. Suzuki, N. J. Avdi, K. C. Malcolm, and G. S. Worthen, "Lipopolysaccharide-induced c-Jun NH2terminal kinase activation in human neutrophils: role of phosphatidylinositol 3-kinase and Syk-mediated pathways," Journal of Biological Chemistry, vol. 279, no. 12, pp. 1088310891, 2004.

[33] C. Olsnes, T. Bredholt, J. Olofsson, and H. J. Aarstad, "OK-432-stimulated chemokine secretion from human monocytes depends on MEK1/2, and involves p38 MAPK and NF- $\kappa$ B phosphorylation, in vitro," APMIS, vol. 121, no. 4, pp. 299-310, 2013.

[34] Y. Q. Lu, S. Zhong, N. Meng, Y. P. Fan, and W. X. Tang, "NLRP3 inflammasome activation results in liver inflammation and fibrosis in mice infected with Schistosoma japonicum in a Syk-dependent manner," Scientific Reports, vol. 7, no. 1, p. 8120, 2017.

[35] E. B. Duffy, S. Periasamy, D. Hunt, J. R. Drake, and J. A. Harton, "Fc $\gamma$ R mediates TLR2- and Syk-dependent NLRP3 inflammasome activation by inactivated Francisella tularensis LVS immune complexes," Journal of Leukocyte Biology, vol. 100, no. 6, pp. 1335-1347, 2016.

[36] D. Patel, S. Gaikwad, N. Challagundla, M. Nivsarkar, and R. Agrawal-Rajput, "Spleen tyrosine kinase inhibition ameliorates airway inflammation through modulation of NLRP3 inflammosome and Th17/Treg axis," International Immunopharmacology, vol. 54, pp. 375-384, 2018.

[37] J. Hirahashi, D. Mekala, J. van Ziffle et al., "Mac-1 signaling via Src-family and Syk kinases results in elastase-dependent thrombohemorrhagic vasculopathy," Immunity, vol. 25, no. 2, pp. 271-283, 2006.

[38] X.-l. Sun, B. Y.-K. Law, I. R. de Seabra Rodrigues Dias, S. W. F. Mok, Y.-z. He, and V. K.-W. Wong, "Pathogenesis of thromboangiitis obliterans: gene polymorphism and immunoregulation of human vascular endothelial cells," Atherosclerosis, vol. 265, pp. 258-265, 2017.

[39] B. A. Bouchard and P. B. Tracy, "Platelets, leukocytes, and coagulation," Current Opinion in Hematology, vol. 8, no. 5, pp. 263-269, 2001.

[40] J. Brozna, "Shwartzman reaction," Seminars in Thrombosis and Hemostasis, vol. 16, no. 4, pp. 326-332, 1990.

[41] O. Gasser and J. A. Schifferli, "Microparticles released by human neutrophils adhere to erythrocytes in the presence of complement," Experimental Cell Research, vol. 307, no. 2, pp. 381-387, 2005.

[42] S. S. Ketha and L. T. Cooper, "The role of autoimmunity in thromboangiitis obliterans (Buerger's disease)," Annals of the New York Academy of Sciences, vol. 1285, no. 1, pp. 15-25, 2013.

[43] Z. Wei, W. Jiang, H. Wang et al., "The IL-6/STAT3 pathway regulates adhesion molecules and cytoskeleton of endothelial cells in thromboangiitis obliterans," Cellular Signalling, vol. 44, pp. 118-126, 2018.

[44] S. Wu, X. Sun, W. Wu, D. Shi, and T. Jiang, "Effect of revascularization on IL- 6 and TNF- $\alpha$ in patients with thromboangiitis obliterans," Experimental and Therapeutic Medicine, vol. 15, no. 4, pp. 3947-3951, 2018.

[45] J. Narváez, C. García-Gómez, L. Álvarez et al., "Efficacy of bosentan in patients with refractory thromboangiitis obliterans (Buerger disease): a case series and review of the literature," Medicine, vol. 95, no. 48, article e5511, 2016. 


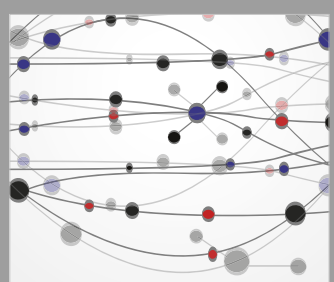

The Scientific World Journal
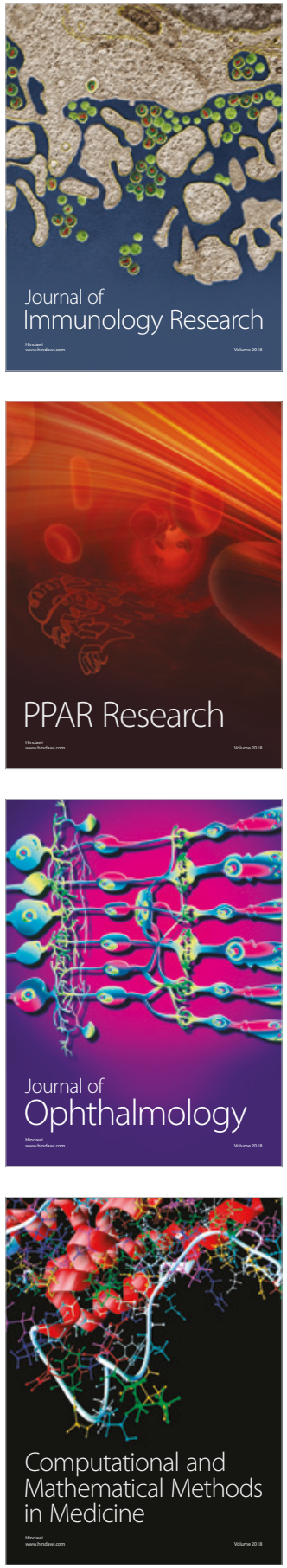

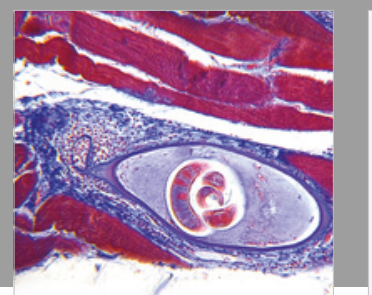

Gastroenterology Research and Practice

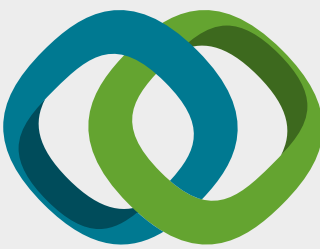

\section{Hindawi}

Submit your manuscripts at

www.hindawi.com
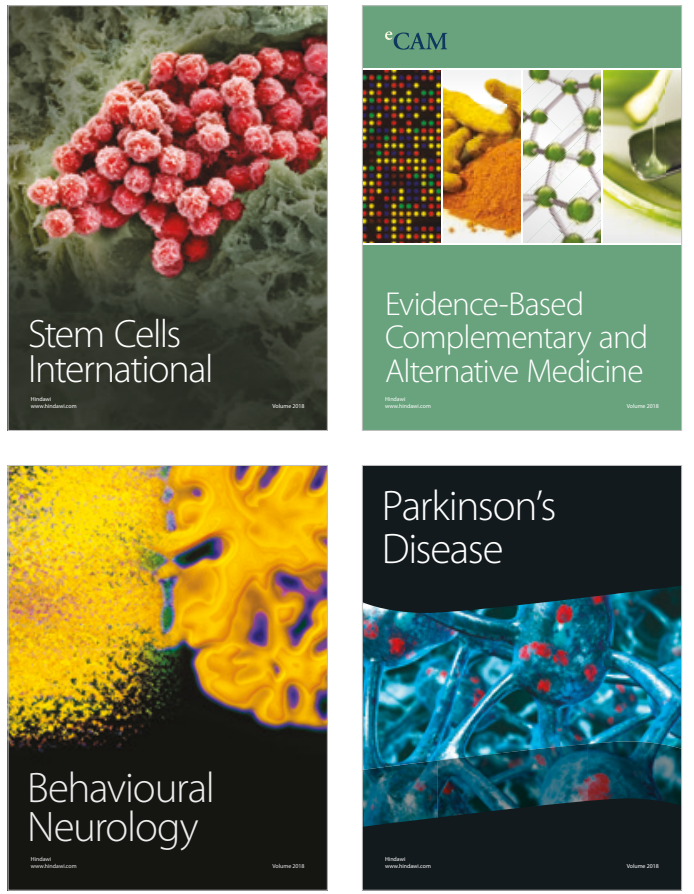

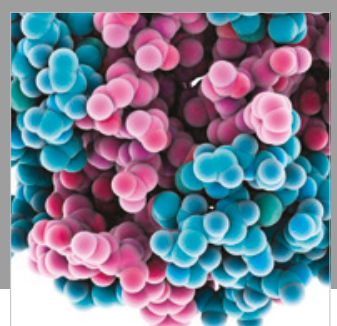

ournal of

Diabetes Research

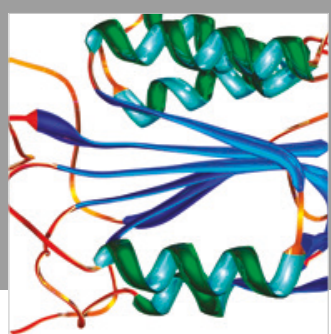

Disease Markers
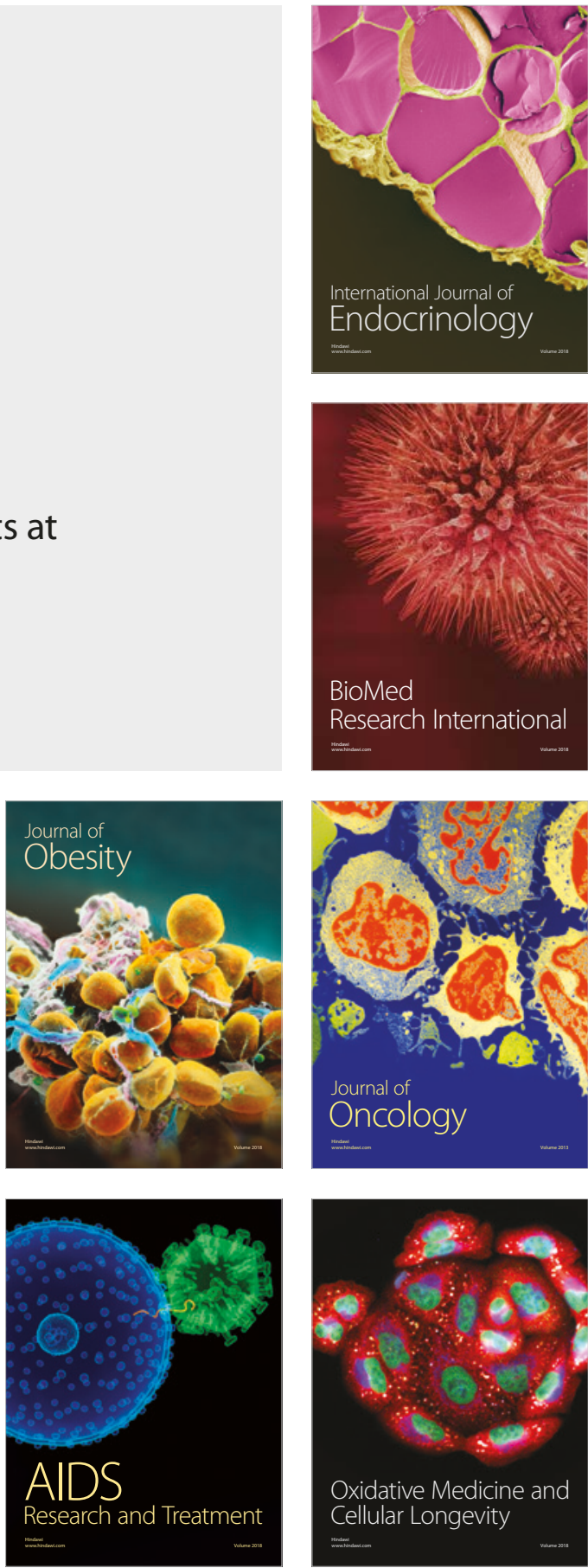